\title{
Hyperboloidal foliations and scri-fixing
}

\author{
Anıl Zenginoğlu \\ Max-Planck-Institut für Gravitationsphysik, Albert-Einstein-Institut, Am Mühlenberg 1, \\ D-14476 Golm, Germany \\ E-mail: anil@aei.mpg.de
}

Received 15 February 2008, in final form 15 April 2008

Published 19 June 2008

Online at stacks.iop.org/CQG/25/145002

\begin{abstract}
A useful choice of gauge when including null infinity in the computational domain is scri-fixing, that is, fixing the spatial coordinate location of null infinity. This choice allows us to avoid the introduction of artificial timelike outer boundaries in numerical calculations. We construct manifestly stationary scri-fixing coordinates explicitly on Minkowski, Schwarzschild and Kerr spacetimes.
\end{abstract}

PACS numbers: 04.20.Dm, 04.25.Ha, 04.20.Jb

\section{Motivation}

In numerical studies of the initial value problem for test fields on asymptotically flat background spacetimes, one typically truncates the solution domain by introducing an artificial timelike outer boundary into the spacetime. The solution then is calculated on a finite, spatially compact domain. The boundary of this domain is not part of the physical problem. Therefore, one tries to construct boundary conditions that correspond to transparency of this artificial outer boundary. In addition, these boundary conditions are required to form a well-posed initial boundary value problem (IBVP).

In general, it is not possible to construct such boundary conditions. Spurious reflections occur from the outer boundary even for the simple case of the flat wave equation on a threedimensional ball [50]. One therefore tries to minimize the amount of such spurious reflections in a manner that also ensures the well-posedness of the IBVP. Such boundary conditions are called non-reflecting or absorbing. It is, however, very difficult to construct them when the curvature of the background does not vanish or when nonlinear terms appear in the equations. One needs to account for backscatter off curvature or self-interaction of the field near the boundary. A bad choice of boundary data can destroy relevant features of the solution. It has been shown, for example, that a certain choice of boundary data, commonly used in numerical relativity, destroys the polynomial tail behavior of solutions to wave equations on a Schwarzschild spacetime [8]. 
A further difficulty is related to the notion of radiation. In the study of radiative phenomena, one is interested in the behavior of solutions in the far-field zone. The electromagnetic field on flat space, for example, admits a global separation into near-field terms and far-field terms which simplifies the discussion of radiation considerably. When the background curvature does not vanish, such a separation can only be expected in the asymptotic limit to infinity in null directions. Therefore to study radiation accurately, one needs to calculate very large portions of spacetime. Using Cauchy-type foliations and truncating the computational domain, however, does not allow us to calculate such large portions of spacetime that astrophysically realistic distances can be modeled in a numerical calculation.

Additional complications arise when one tries to construct absorbing boundary conditions for the Einstein equations [7, 19, 48, 51]. The treatment of the asymptotic region in numerical relativity is an important theoretical and practical problem as it effects the accuracy of the calculated radiation field [41, 49]. With increasing sensitivity of detectors and computer power, systematic errors in current numerical simulations due to wave extraction may restrict the predictable power of numerical relativity and blur the comparison between observed and calculated waveforms [5, 30].

A clean solution to the above mentioned difficulties is available on the level of geometry. The solution is to include null infinity in the computational domain. This geometric idea goes back to Penrose and has been known for a long time $[42,43]$. Its implementation within the $3+1$ approach, however, has been exceptionally difficult, numerically as well as analytically. In this paper, we discuss in detail the construction of suitable explicit gauges on asymptotically flat background spacetimes such that the geometric idea of null infinity can be incorporated in numerical calculations within the $3+1$ approach. While we assume the spacetime to be given, a basic motivation is to extend the suggested methods to the treatment of the asymptotic region in numerical solutions to the Einstein equations.

The paper is organized as follows: in section 2 we introduce the basic concepts and explain the idea of scri-fixing. In section 3, we present an explicit construction of scrifixing coordinates in spherical symmetry. This method is applied to Minkowski spacetime in section 4 and to extended Schwarzschild spacetime in section 5. Going beyond spherical symmetry, we present the construction of a scri-fixing gauge on Kerr spacetime in section 6 . We conclude in section 7 with a discussion of scri-fixing and an outlook for future work.

\section{The general idea}

If a background spacetime $(\widetilde{\mathcal{M}}, \tilde{g})$ is asymptotically flat in a suitable sense, one can attach to the spacetime manifold $\widetilde{\mathcal{M}}$ a boundary in null directions, $\mathscr{I}$, such that a certain conformal rescaling of the physical metric $\tilde{g}$ results in a metric $g=\Omega^{2} \tilde{g}$ with a smooth extension through that boundary and the conformal factor $\Omega$ has a specified behavior on that boundary. In particular, we require $\left.\Omega\right|_{\widetilde{\mathcal{M}}}>0,\left.\Omega\right|_{\mathscr{I}}=0$ and $\left.\mathrm{d} \Omega\right|_{\mathscr{I}} \neq 0$. The extended manifold with boundary is denoted by $\mathcal{M}$. We call the triple $(\mathcal{M}, g, \Omega)$ a conformal extension and say that the spacetime $(\widetilde{\mathcal{M}}, \tilde{g})$ is weakly asymptotically simple. All known explicit solutions to the Einstein equations that may be considered as 'physically reasonable' and asymptotically flat are weakly asymptotically simple [31].

By construction, null rays reach $\mathscr{I}$ for an infinite value of the affine parameter along them. Therefore we refer to $\mathscr{I}$ as null infinity. It turns out, under reasonable conditions, that $\mathscr{I}$ consists of two parts $\mathscr{I}^{+}$and $\mathscr{I}^{-}$, called future and past null infinity respectively, each with topology $\mathbb{R} \times S^{2}$ [43]. We will mainly focus on future null infinity.

The propagation of massless fields is governed by the null cone structure of spacetime. It is therefore convenient in studies of radiative properties of test fields to choose a foliation 
that is closely related to outgoing null directions. An option is to choose a characteristic foliation. This approach has been very successful in situations where null foliations cover the computational domain in a regular fashion $[2,23,25,36,53]$. The main difficulty with this approach can be traced back to the fact that characteristic foliations are not well behaved in regions of strong dynamical gravitational fields due to the formation of caustics in the bundles of light rays generating the null hypersurfaces [20].

Spacelike foliations are more flexible than characteristic ones. It turns out that spacelike surfaces can be constructed that extend through null infinity. Such surfaces are called hyperboloidal as their asymptotic behavior is similar to standard hyperboloids in Minkowski spacetime [17]. Instead of approaching spatial infinity as Cauchy surfaces do, they reach null infinity, $\mathscr{I}$, which makes them suitable for radiation extraction. It should be emphasized that hyperboloidal surfaces extend through null infinity as spacelike surfaces. It would be misleading to refer to them as asymptotically null because their tangent vector never becomes null. The term asymptotically null may be reserved for surfaces that become null at or close to null infinity, in which case, for example, their mean extrinsic curvature would be unbounded.

One can formulate a Cauchy problem based on hyperboloidal surfaces [17, 22]. Although the hyperboloidal initial value problem and its merits have been known for a long time, numerical studies of test fields based on hyperboloidal foliations are rare. One major difficulty is related to the choice of gauge. Future null infinity is an ingoing null surface. This property can be made manifest in the gauge such that null generators of $\mathscr{I}^{+}$converge. Such coordinates can be useful in numerical studies for discussing global properties of spacetimes, for example with respect to the investigation of a point corresponding to timelike infinity $i^{+}[33,55]$. These coordinates, however, do not seem to be convenient for calculating radiation along $\mathscr{I}^{+}$, as they imply a resolution loss in the physical part of the conformal extension and require a gauge and boundary treatment in the unphysical part. We emphasize that, as also follows from abstract, semi-global existence results [18], the resolution loss is not an intrinsic deficiency of conformal compactification, but a consequence of a bad choice of gauge for the type of problem that one is interested in.

A suitable class of gauges on hyperboloidal foliations for studying radiation is given by scri-fixing [16]. In such a gauge the spatial coordinate location of null infinity is independent of the time coordinate so that $\mathscr{I}^{+}$is represented by the same grid points during time evolution and no resolution loss appears. This allows us to calculate radiation emitted to future null infinity in a very accurate way using a simple extraction procedure along an a priori known grid surface. Further, one can let the numerical outer boundary coincide with $\mathscr{I}^{+}$so that no boundary treatment on the continuum level is required [35]. It should be noted that in a characteristic approach based on a double null gauge, scri-fixing is automatically satisfied by the coordinates. This seems to be one of the key advantages in including $\mathscr{I}^{+}$in the computational domain within the characteristic approach. Within the hyperboloidal approach, the larger freedom of gauge choices needs to be carefully dealt with to achieve scri-fixing in the asymptotic domain.

The first explicit construction of a scri-fixing gauge has been given for Minkowski spacetime [34, 39]. Its usefulness has been demonstrated in studies of dynamical magnetic monopoles on a flat background $[14,15]$. The explicit constructions presented in this paper should be useful for studies of test fields on black hole spacetimes [54]. They are also intended to provide examples for testing ideas on treating the hyperboloidal initial value problem for the Einstein equations as well as for constructing hyperboloidal initial data $[1,56]$.

A convenient way to discuss global properties of foliations is to depict their embedding in Penrose diagrams. We will employ such diagrams in this paper to plot results of calculations. 
In spherical symmetry, Penrose diagrams accurately represent the global causal structure of the spacetime under study (see [9] for a rigorous discussion of Penrose diagrams).

\section{Scri-fixing in spherical symmetry}

The main property of a scri-fixing gauge is that the zero set of the conformal factor in terms of local coordinates is independent of time. There are two steps involved in the construction of such a gauge: the choice of a hyperboloidal foliation and a conformal compactification, that is, the introduction of compactifying spatial coordinates in combination with a suitable conformal factor. We present in the following how these steps can be carried out explicitly for asymptotically flat, spherically symmetric spacetimes. The techniques can be easily carried over to spacetimes with less symmetries as demonstrated in section 6.

\subsection{Hyperboloidal foliations}

Assume that we are given a globally hyperbolic, asymptotically flat, spherically symmetric spacetime $(\widetilde{\mathcal{M}}, \tilde{g})$ so that the group $S O(3)$ acts non-trivially by isometry on $(\widetilde{\mathcal{M}}, \tilde{g})$. We can introduce on the quotient space $\widetilde{\mathcal{Q}}=\widetilde{\mathcal{M}} / S O(3)$ a natural function $\tilde{r}: \widetilde{\mathcal{Q}} \rightarrow \mathbb{R}$, called the area radius, such that the group orbits of points $p \in \widetilde{\mathcal{Q}}$ have area $4 \pi \tilde{r}(p)^{2}$. The metric $\tilde{g}$ on $\widetilde{\mathcal{M}}$ can then be written as $\tilde{g}=\tilde{q}+\tilde{r}^{2} \mathrm{~d} \sigma^{2}$, where $\mathrm{d} \sigma^{2}$ is the standard metric on $S^{2}$ and $\tilde{q}$ is a Lorentzian metric of rank 2 that descends naturally to a metric on $\widetilde{\mathcal{Q}}$. Due to the Jebsen-Birkhoff theorem [37], we can assume that, beside the $S O$ (3) generators, there is an additional hypersurface orthogonal Killing vector field on $\widetilde{\mathcal{M}}$ that is timelike in the exterior [11]. We can introduce a time function $\tilde{t}$ such that this Killing vector field is given by $\partial_{\tilde{t}}$ and level sets of $\tilde{t}$ are orthogonal to it. In these coordinates, the spacetime metric reads

$$
\tilde{g}=\tilde{q}_{\tilde{t} \tilde{t}} \mathrm{~d} \tilde{t}^{2}+\tilde{q}_{\tilde{r} \tilde{r}} \mathrm{~d} \tilde{r}^{2}+\tilde{r}^{2} \mathrm{~d} \sigma^{2},
$$

where $\tilde{q}_{\tilde{t} \tilde{t}}$ and $\tilde{q}_{\tilde{r} \tilde{r}}$ are functions of $\tilde{r}$ only. The time function $\tilde{t}$ is by construction such that the time slices are Cauchy surfaces and approach spatial infinity in the asymptotic region. We are interested in null infinity. Therefore, we need to introduce a new coordinate $t=t(\tilde{t}, \tilde{r})$ such that the time slices are hyperboloidal surfaces.

In stationary spacetimes one may define the notion of Killing observers. The worldline of a Killing observer corresponds to the integral curves of the timelike Killing field. These observers are not inertial if the curvature of the spacetime does not vanish, but they are natural in the sense that their distance to each other remains constant. A Killing observer far away from a source can be regarded as representing a gravitational wave detector. Therefore, we wish to keep the natural coordinate representation of the Killing observers so that the Killing vector field is given by $\partial_{t}$ in terms of the new coordinate $t$. This implies a transformation of the form

$$
t=\tilde{t}-h(\tilde{r}),
$$

where $h(\tilde{r})$ is called the height function. In this new time coordinate, the quotient metric $\tilde{q}$ can be written as

$$
\tilde{q}=\tilde{q}_{\tilde{t} \tilde{t}} \mathrm{~d} t^{2}+2 \tilde{q}_{\tilde{t} \tilde{t}} h^{\prime} \mathrm{d} t \mathrm{~d} \tilde{r}+\left(\tilde{q}_{\tilde{t} \tilde{t}} h^{\prime 2}+\tilde{q}_{\tilde{r} \tilde{r}}\right) \mathrm{d} \tilde{r}^{2}, \quad \text { where } \quad h^{\prime}:=\frac{\mathrm{d} h}{\mathrm{~d} \tilde{r}} .
$$

All metric components are functions of $\tilde{r}$ only. A major advantage of this manifest time independence of the metric functions is the implied simplification in numerical applications.

The next question is how to choose the height function to obtain a hyperboloidal foliation. As the time slices are required to be spacelike and to approach future null infinity, the height 
function should lead to a 'bending up' of the surfaces as $\tilde{r} \rightarrow \infty$ such that, asymptotically, their behavior is similar to outgoing null surfaces. In the following subsection we will derive a necessary requirement on the asymptotic behavior of the height function for the regularity of the conformal compactification.

\subsection{Scri-fixing conformal compactification}

On level sets of the hyperboloidal time function $t$ we introduce a compactifying coordinate $r$. The physical metric $\tilde{g}$ then becomes singular at the domain boundary of the compactifying coordinate. To compensate for this singular behavior we rescale the metric with a conformal factor $\Omega$ that vanishes at that boundary in a suitable way. This procedure is referred to as conformal compactification.

It is natural and convenient to require that the compactifying coordinate $r$ is an area radius on the conformal extension with respect to the rescaled metric $g=\Omega^{2} \tilde{g}$. This requirement implies $\tilde{r}=r / \Omega$. The zero set of the conformal factor corresponds to the asymptotic region of the physical spacetime. Writing the rescaled quotient metric $q=\Omega^{2} \tilde{q}$ in terms of the compactifying coordinate $r$ using $\tilde{r}(r)=r / \Omega(r)$, we get

$$
q=\Omega^{2} \tilde{q}_{\tilde{t} \tilde{t}} \mathrm{~d} t^{2}+2 \tilde{q}_{\tilde{t} \tilde{t}} h^{\prime}\left(\Omega-\Omega^{\prime} r\right) \mathrm{d} t \mathrm{~d} r+\frac{\tilde{q}_{\tilde{t} \tilde{t}} h^{\prime 2}+\tilde{q}_{\tilde{r} \tilde{r}}}{\Omega^{2}}\left(\Omega-\Omega^{\prime} r\right)^{2} \mathrm{~d} r^{2}
$$

This formula can be applied with almost any choice of conformal factor. Note that we have some freedom here. We can require, for example, that $r$ be the proper distance in the conformal extension, i.e. $q_{r r}=1$. Then the conformal factor is determined by this requirement and cannot, in general, be written in an explicit form [40]. In contrast, by allowing the metric component $q_{r r}$ to be more general, we are free to prescribe the representation of a conformal factor in terms of a suitable compactifying radial coordinate $r$ and the coordinate transformation is explicit.

A convenient representation for the conformal factor that we will resort to is $\Omega=(1-r)$. Our discussion is not restricted by this choice. Other coordinate representations of the conformal factor are also allowed. The above choice is not good at the origin as the gradient of $r$ vanishes at this point. A suitable representation can be used in the interior as will be discussed in the following subsection. The above choice fixes the compactifying coordinate $r$ via

$$
\tilde{r}(r)=\frac{r}{1-r}
$$

implying

$$
q=\Omega^{2} \tilde{q}_{\tilde{t} \tilde{t}} \mathrm{~d} t^{2}+2 \tilde{q}_{\tilde{t} \tilde{t}} h^{\prime} \mathrm{d} t \mathrm{~d} r+\frac{\tilde{q}_{\tilde{t} \tilde{t}} h^{\prime 2}+\tilde{q}_{\tilde{r} \tilde{r}}}{\Omega^{2}} \mathrm{~d} r^{2} .
$$

The vanishing of $q_{t t}$ at $\{\Omega=0\}$ implies that $\partial_{t}$ becomes null at this surface. For regularity of the metric we require

$$
h^{\prime}=\sqrt{\frac{C \Omega^{2}-\tilde{q}_{\tilde{r} \tilde{r}}}{\tilde{q}_{\tilde{t} \tilde{t}}}} \quad \text { as } \quad \tilde{r} \rightarrow \infty, \quad \text { with } \quad C=\text { const. }
$$

To gain an intuition for the behavior of hyperboloidal surfaces, we will calculate coordinate speeds of characteristics. The coordinate speed $c$ of ingoing and outgoing null rays in radial directions can be calculated by $c_{\mp}=\left(-q_{t r} \pm \sqrt{q_{t r}^{2}-q_{r r} q_{t t}}\right) / q_{r r}$. The speeds are independent of the conformal rescaling of the metric which is a reflection of the fact that conformal rescaling leaves the null cone structure invariant. The positive sign on the right-hand side corresponds to ingoing null rays, the negative sign corresponds to outgoing null rays. On the conformal 
boundary we get $c_{\mp}=\left(-q_{t r} \pm q_{t r}\right) / q_{r r}$. As expected, the ingoing characteristic speed is zero. Consequently no analytic boundary treatment is needed or allowed. The outgoing characteristic speed is $\left.c_{+}\right|_{\mathscr{I}^{+}}=-2 q_{t r} / q_{r r}$. In a future hyperboloidal foliation we have $q_{t r} \mid \mathscr{I}^{+}<0$ and $q_{r r}>0$, so that $c_{+}{\mid \mathscr{I}^{+}}>0$ and radiation can leave the spacetime through future null infinity.

\subsection{Matching}

An important feature of hyperboloidal surfaces, in contrast to characteristic ones, is that their distinctive feature is determined in the asymptotic region. While being spacelike or null is a local property, being hyperboloidal or Cauchy is a global property. This fact allows us to construct useful hyperboloidal foliations easily by a matching procedure.

Foliations commonly used in the $3+1$ approach are characterized by properties which imply that the hypersurfaces approach spatial infinity in the asymptotic domain. One may wish to keep these properties in the interior and transform the surfaces in the exterior such that they approach null infinity asymptotically. Hyperboloidal foliations can be constructed that coincide with arbitrary spacelike foliations in the interior. To achieve this, we will employ the following smooth transition between interior and exterior height functions denoted by $h_{i}$ and $h_{e}$, respectively

$h(\tilde{r})= \begin{cases}h_{i}(\tilde{r}) & \text { for } \tilde{r} \leqslant \tilde{r}_{i}, \\ \mathrm{e}^{-\left(\tilde{r}-\tilde{r}_{i}\right)^{2} /\left(\tilde{r}-\tilde{r}_{e}\right)^{2}}\left(h_{i}(\tilde{r})-h_{e}(\tilde{r})\right)+h_{e}(\tilde{r}) & \text { for } \tilde{r}_{i}<\tilde{r}<\tilde{r}_{e}, \\ h_{e}(\tilde{r}) & \text { for } \tilde{r} \geqslant \tilde{r}_{a} .\end{cases}$

The height function needs to satisfy $\left|h^{\prime}\right|<\sqrt{\tilde{q}_{\tilde{r} \tilde{r}} /\left(-\tilde{q}_{\tilde{t} \tilde{t}}\right)}$ for the foliation to be spacelike. We may apply the above transition also with respect to derivatives of the corresponding height functions. We may set the conformal factor to unity in the interior with a smooth transition to its asymptotic representation in the exterior. The transition domain $\left(\tilde{r}_{i}, \tilde{r}_{e}\right)$ should be in the exterior, but not necessarily in the far-field zone. The free parameters of the matching can be decided upon by numerical experiments. Detailed examples will be discussed in later sections.

One should note that this matching is fundamentally different from Cauchy-characteristic matching where one matches a spacelike and a null surface. The matching in our case results in a single, smooth, hyperboloidal surface with an arbitrary behavior in the interior determined by $h_{i}(\tilde{r})$.

\section{Minkowski spacetime}

\subsection{Cauchy foliation}

The two-dimensional Minkowski metric $\tilde{\eta}$ on the quotient space in standard coordinates is given by $\tilde{\eta}=-\mathrm{d} \tilde{t}^{2}+\mathrm{d} \tilde{r}^{2}$. The Penrose diagram in figure 1 depicts the causal structure of Minkowski spacetime. Each point in the diagram represents a sphere except the point $i^{0}$ that corresponds to spatial infinity and the points along the vertical line segment connecting $i^{+}$and $i^{-}$that correspond to the origin $\{\tilde{r}=0\}$. Radial null surfaces are represented by straight line segments with $45^{\circ}$ to the horizontal. Curves with a smaller angle represent spacelike surfaces, curves with a larger angle represent timelike surfaces. Level surfaces of $\tilde{r}$ approach timelike infinity denoted by $i^{ \pm}$, level surfaces of $\tilde{t}$ approach spatial infinity denoted by $i^{0}$.

It is evident from figure 1 that an outgoing null ray never leaves the surfaces of a Cauchy foliation. This is demonstrated more clearly by the characteristics with respect to coordinates 


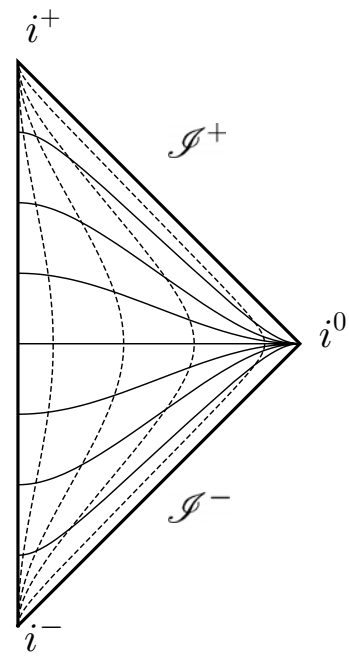

Figure 1. Penrose diagram of Minkowski spacetime. Solid lines represent $\tilde{t}=$ const. surfaces, dashed lines represent $\tilde{r}=$ const. surfaces.

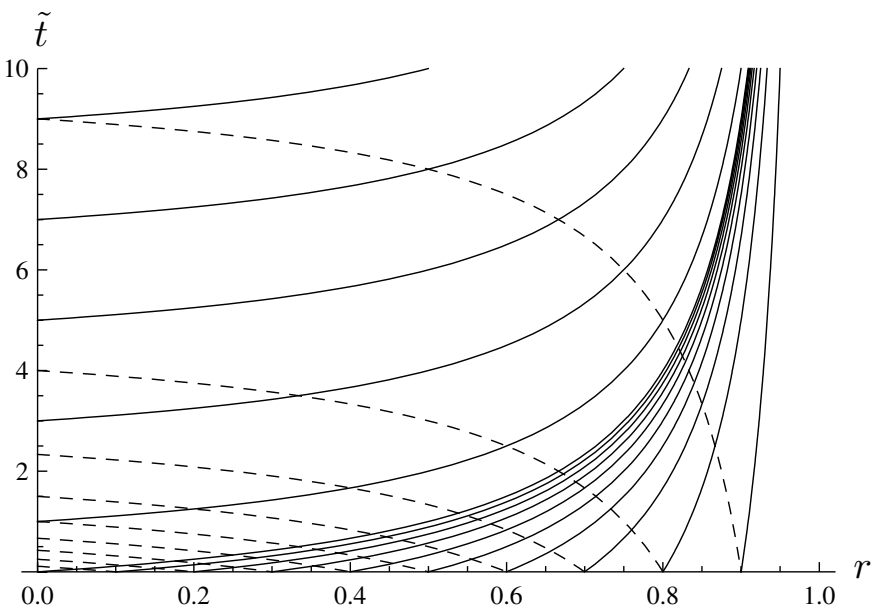

Figure 2. Characteristics in Minkowski spacetime in the standard foliation with respect to the compactifying coordinate $r$ defined in (3). In- and outgoing null surfaces are represented by dashed and solid lines, respectively. Note the different scales in $\tilde{t}$ and $r$.

of the standard foliation. We introduce on the Cauchy surfaces the compactifying coordinate $r$ defined in (3). The resulting metric reads $\tilde{\eta}=-\mathrm{d} \tilde{t}^{2}+\mathrm{d} r^{2} /(1-r)^{4}$, and the coordinate speeds of characteristics become $c_{ \pm}= \pm(1-r)^{2}$. The characteristic speeds decrease to zero in the asymptotic region close to $r=1$. In simulations, this results in a slowing down of outgoing waves. This feature is clearly shown in figure 2 where in- and outgoing characteristics are depicted by dashed and solid lines, respectively. We see that outgoing characteristics pile up during the evolution near the boundary and eventually, cannot be resolved (see [45] for a discussion of this phenomena in the context of a binary black hole evolution). 


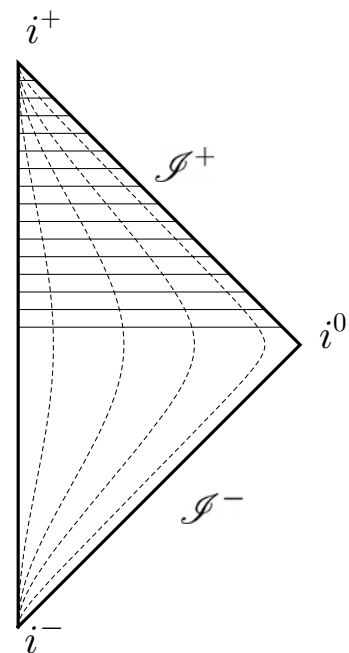

Figure 3. Penrose diagram of a hyperboloidal foliation of Minkowski spacetime. Solid lines represent hyperboloidal surfaces, dashed lines represent Killing observers.

A suitable compactified foliation should not only have no ingoing characteristics coming in from the outer boundary, but also allow outgoing characteristics to leave the simulation domain.

\subsection{Hyperboloidal foliations}

A typical example for a future hyperboloidal foliation is plotted in Penrose diagram figure 3. Null rays do leave the surfaces of a hyperboloidal foliation. The corresponding time function to the foliation in figure 3 is given for $\tilde{t}>0$ by

$$
t(\tilde{t}, \tilde{r})=\frac{1}{2 \tilde{t}}\left(\tilde{t}^{2}-\tilde{r}^{2}-1\right)
$$

A distinctive feature of hyperboloidal surfaces is that their mean extrinsic curvature attains a non-vanishing, finite value at infinity. We use the convention in which positive $\tilde{K}$ corresponds to expansion so that positive asymptotic values of $\tilde{K}$ correspond to future hyperboloidal surfaces. The mean extrinsic curvature of level sets of $t$ as defined in (7) reads $\tilde{K}=3 / \sqrt{1+t^{2}}$. Each surface has a spatially constant mean curvature. The foliation, however, does not seem to be convenient for numerical calculations, because the time function does not have the form (2). The Minkowski metric in this new coordinate depends on time.

A more convenient foliation can be constructed by Lie dragging a standard hyperboloid along the timelike Killing field. The spherically symmetric standard hyperboloids in Minkowski spacetime are given by $a^{2}=\tilde{t}^{2}-\tilde{r}^{2}$, with $a \in \mathbb{R}_{+}$. Translating these surfaces along $\partial_{\tilde{t}}$ by an amount of $t$, we get the equation $a^{2}=(\tilde{t}-t)^{2}-\tilde{r}^{2}$. The new time coordinate $t$ is then defined by

$$
t(\tilde{t}, \tilde{r})=\tilde{t}-\sqrt{a^{2}+\tilde{r}^{2}} .
$$

Each surface of the foliation has a constant mean curvature $\tilde{K}=3 / a$. The Penrose diagram in figure 4 shows foliations defined by (8) for different values of $\tilde{K}$. 

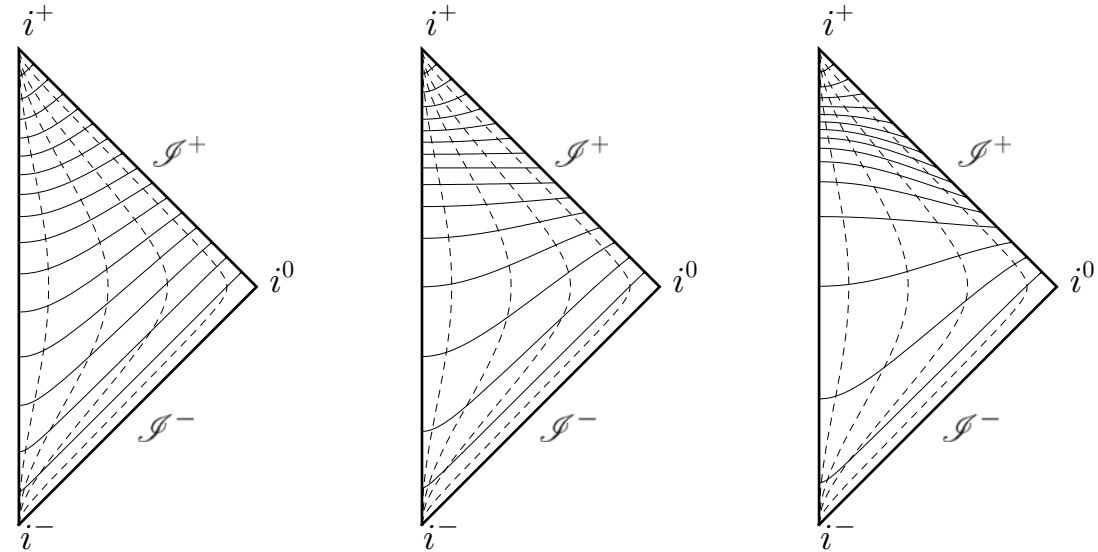

Figure 4. Penrose diagrams for constant mean curvature (CMC) foliations of Minkowski spacetime. Solid lines represent hyperboloidal surfaces, dashed lines represent Killing observers. The foliations have $\tilde{K}=\{6,3,2\}$ from left to right.
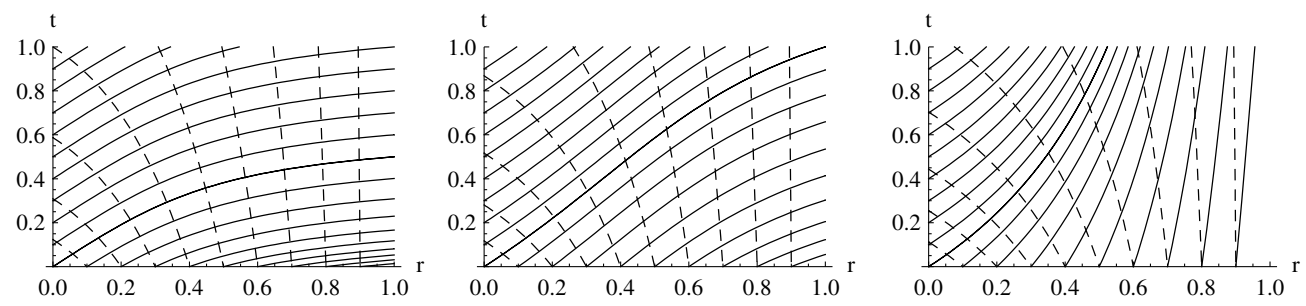

Figure 5. Coordinate representations of in- and outgoing characteristics of (9) with $\tilde{K}=3 / a=$ $\{6,3,0.5\}$. High values for $\tilde{K}$ are seen to be restrictive in a numerical calculation in which the numerical cone needs to be larger than the characteristic cone.

In this new time coordinate, scri-fixing conformal compactification as in (4) results in

$$
\eta=-\Omega^{2} \mathrm{~d} t^{2}-\frac{2 r}{\sqrt{a^{2} \Omega^{2}+r^{2}}} \mathrm{~d} t \mathrm{~d} r+\frac{a^{2}}{a^{2} \Omega^{2}+r^{2}} \mathrm{~d} r^{2} .
$$

We see from the Penrose diagram in figure 4 that the mean extrinsic curvature of the surfaces determines the angle at which the surfaces cut null infinity. It also acts as a measure for how close the surfaces are to outgoing null surfaces. The similarity of future hyperboloidal surfaces to outgoing null surfaces can be quantified by the coordinate speed of the outgoing characteristic at null infinity. We have $\left.c_{+}\right|_{\mathscr{I}^{+}}=4 \tilde{K}^{2} / 9$. A large value of $\tilde{K}$ corresponds to surfaces which are closer to null surfaces, as also suggested by the Penrose diagrams in figure 4. This is seen more clearly in figure 5, where in- and outgoing characteristics have been plotted with respect to coordinates. Note that the choice of a high value for $\tilde{K}$ in a numerical calculation using an explicit time integration algorithm would require very small time steps due to the Courant condition which demands that the numerical cone needs to be larger than the characteristic cone. For small values of $\tilde{K}$, however, this condition can be easily satisfied as seen also in figure 5 .

It should be emphasized that the notion of 'proximity' to null surfaces does not have a general geometric meaning. Geometrically, it makes no sense to claim that a spacelike surface 

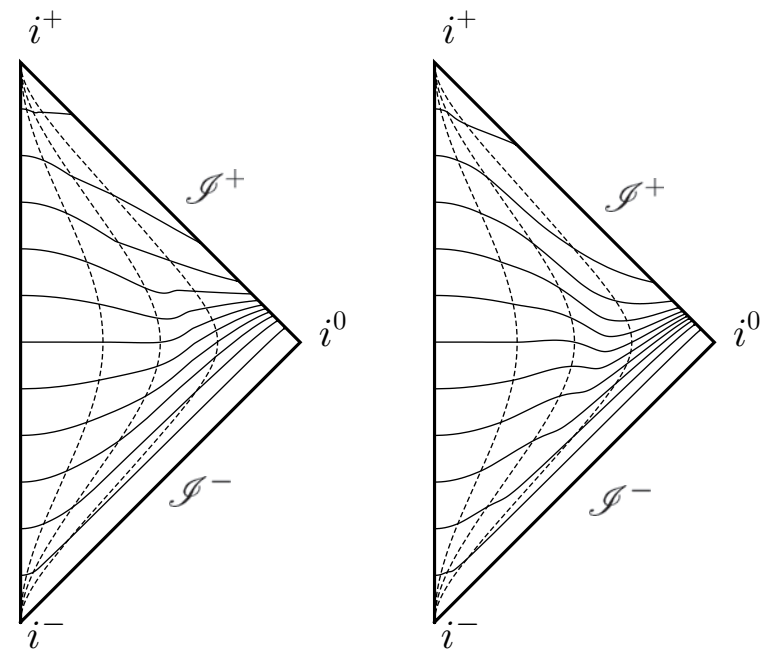

Figure 6. Transition between maximal surfaces and two types of hyperboloidal surfaces determined by (10) and (11). The three dashed lines depict the timelike surfaces $\tilde{r}_{i}=1 / 2, \tilde{r}_{m}=1$, and $\tilde{r}_{e}=2$. The exterior foliation has $a=2$ in both cases.

is close to a null surface as this depends on the chosen frame of reference. On the other hand, in stationary spacetimes, we have a natural choice of observers, namely the Killing observers, so that we can introduce such a notion. In the general non-stationary case, this notion may be useful in the asymptotic domain with respect to the asymptotic Killing observers.

\subsection{Matching}

Level surfaces of the standard time coordinate $\tilde{t}$ in Minkowski spacetime are maximal, i.e. their mean extrinsic curvature vanishes. To match an interior maximal foliation to a hyperboloidal foliation, we choose in (6)

$$
h_{i}=C_{i}, \quad h_{e}=C_{e}+\sqrt{a^{2}+\tilde{r}^{2}} .
$$

The constants $C_{i}$ and $C_{e}$ must be chosen such that the matched surface is spacelike in the transition domain, that is, $\left|h^{\prime}\right|<1$. One convenient way to achieve this is to require that the surfaces intersect within the transition domain. We denote this matching point by $\tilde{r}_{m} \in\left(\tilde{r}_{i}, \tilde{r}_{e}\right)$, and require $h_{i}\left(\tilde{r}_{m}\right)=h_{e}\left(\tilde{r}_{m}\right)$. This implies $C_{e}=C_{i}-\sqrt{a^{2}+\tilde{r}_{m}^{2}}$. The resulting foliation has been plotted in figure 6 . The parameters have been chosen such that the matching is clearly visible in the diagrams. In a numerical calculation, they need to be decided upon by numerical experience.

A more general way of constructing hyperboloidal surfaces in a given spacetime is to Taylor expand formula (5) near infinity. We get in Minkowski spacetime

$$
h^{\prime}(\tilde{r}) \sim\left(1-\frac{a^{2}}{(1+\tilde{r})^{2}}\right)^{\frac{1}{2}}=1-\frac{a^{2}}{2 \tilde{r}^{2}}+\frac{a^{2}}{\tilde{r}^{3}}+O\left(\frac{1}{\tilde{r}^{4}}\right), \quad \text { as } \quad \tilde{r} \rightarrow \infty,
$$

where we set $C \equiv a^{2}$. The first two terms of the expansion are sufficient to fulfil the requirements on the height function for a future hyperboloidal foliation. The foliation is defined in the exterior domain for $\tilde{r}>a-1$, or written in the compactifying coordinate, for $r>1-1 / a$. We use (6) to match the surfaces to an interior maximal foliation by choosing 


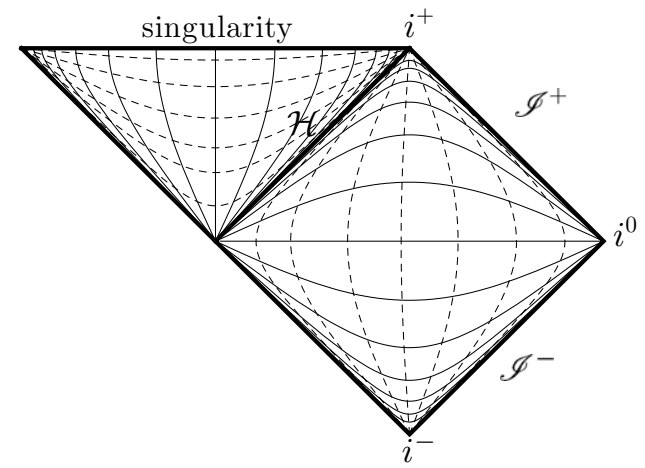

Figure 7. Level sets of Schwarzschild coordinates on the extended Schwarzschild manifold.

$h_{e}(\tilde{r})=\tilde{r}+a^{2} /(2 \tilde{r})+C_{e}$, and $h_{i}=C_{i}$. The resulting foliation has been plotted in figure 6. The rescaled metric in the exterior domain reads

$$
\eta=-\Omega^{2} \mathrm{~d} t^{2}-2\left(1-\frac{a^{2} \Omega^{2}}{2 r^{2}}\right) \mathrm{d} t \mathrm{~d} r+\frac{a^{2}}{2 r^{2}} \mathrm{~d} r^{2} .
$$

The outgoing characteristic speed at future null infinity is $c_{+} \mid \mathscr{I}_{+}=4 / a^{2}$. The mean extrinsic curvature of these surfaces depends on $r$ and attains the value $\tilde{K}=3 / a$ at infinity.

\section{Schwarzschild spacetime}

\subsection{Cauchy-type foliations}

All foliations of Schwarzschild spacetime commonly employed in numerical calculations within the 3+1 approach are Cauchy-type foliations approaching spatial infinity in the asymptotic domain. We mention some of them in this subsection for later reference. We focus on the physically interesting part of the Schwarzschild-Kruskal extension (the regions I and II in [31]) which we refer to as the extended Schwarzschild spacetime.

5.1.1. Schwarzschild coordinates. The two-dimensional Schwarzschild metric in Schwarzschild coordinates is given by

$$
\tilde{q}=-\left(1-\frac{2 m}{\tilde{r}}\right) \mathrm{d} \tilde{t}^{2}+\left(1-\frac{2 m}{\tilde{r}}\right)^{-1} \mathrm{~d} \tilde{r}^{2}, \quad \tilde{r}>2 m .
$$

Level surfaces of $\tilde{t}$ are maximal. They intersect at the bifurcation point and at spatial infinity as seen in figure 7. Therefore, these coordinates are suitable neither to study the region near the black hole, nor the asymptotic domain.

5.1.2. Eddington-Finkelstein coordinates. In numerical applications, a common choice of the so-called 'horizon penetrating' coordinates in Schwarzschild spacetime is defined as in (2) with the height function

$$
h(\tilde{r})=2 m \ln \left|\frac{\tilde{r}}{2 m}-1\right| .
$$




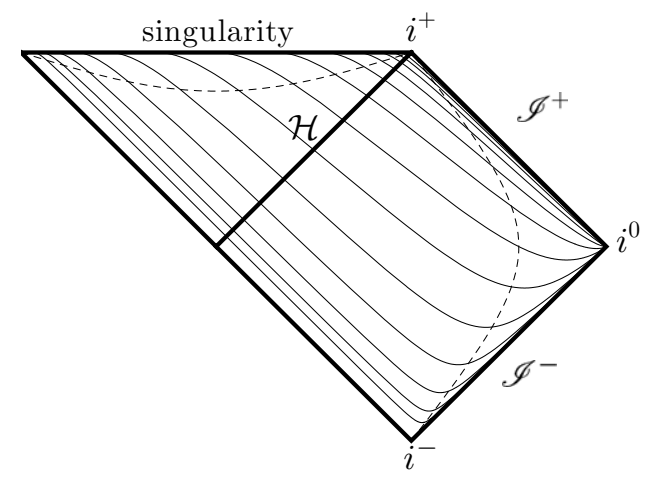

Figure 8. Eddington-Finkelstein foliation suitable for the excision technique. Dashed lines correspond to $\tilde{r}=\{1.5 m, 4 m\}$.

The coordinates such defined have originally been discussed by Eddington [10] and Finkelstein [13]. The Schwarzschild metric on the extended Schwarzschild manifold takes the form

$$
\tilde{q}=-\left(1-\frac{2 m}{\tilde{r}}\right) \mathrm{d} t^{2}+\frac{4 m}{\tilde{r}} \mathrm{~d} t \mathrm{~d} \tilde{r}+\left(1+\frac{2 m}{\tilde{r}}\right) \mathrm{d} \tilde{r}^{2}, \quad \tilde{r}>0 .
$$

The mean extrinsic curvature of level sets of $t$ depends on $\tilde{r}$ and vanishes in the asymptotic domain. The foliation by the time function $t$ is depicted in the Penrose diagram figure 8 . We see that the foliation penetrates the horizon which makes it suitable for excising the singularity from the computational domain. The surfaces are spacelike at each point. They approach spatial infinity in the asymptotic domain which makes them inconvenient to study radiation.

5.1.3. Maximal slices for the puncture technique. Currently, the most popular approach in numerical calculations of black hole spacetimes is the moving puncture technique. The evolution with puncture initial data for a Schwarzschild black hole in a modified $1+\log$ slicing reaches a final stationary state that corresponds to a maximal slice given in suitable coordinates [3, 27-29]. We will use maximal foliations to demonstrate the possibility that hyperboloidal surfaces can be constructed which are suitable for the application of the moving puncture technique in the interior.

The requirement that the mean extrinsic curvature $\tilde{K}$ of the surfaces defined as in (2) are constant implies in Schwarzschild spacetime

$$
\tilde{K}=\frac{1}{\tilde{r}^{2}} \partial_{\tilde{r}}\left(\frac{\tilde{r}^{2} h^{\prime}\left(1-\frac{2 m}{\tilde{r}}\right)}{\sqrt{\left(1-\frac{2 m}{\tilde{r}}\right)^{-1}-h^{\prime 2}\left(1-\frac{2 m}{\tilde{r}}\right)}}\right) .
$$

We can integrate this relation once for a maximal foliation $(\tilde{K}=0)$ in Schwarzschild spacetime to obtain $[4,12]$

$$
h^{\prime}(\tilde{r})=-\frac{C}{\left(1-\frac{2 m}{\tilde{r}}\right) \sqrt{\tilde{r}^{4}-2 m \tilde{r}^{3}+C^{2}}}
$$

where $C$ is a constant of integration. The Schwarzschild metric becomes

$\tilde{q}=-\left(1-\frac{2 m}{\tilde{r}}\right) \mathrm{d} t^{2}+\frac{2 C}{f \tilde{r}^{2}} \mathrm{~d} t \mathrm{~d} \tilde{r}+\frac{1}{f^{2}} \mathrm{~d} \tilde{r}^{2}, \quad$ with $\quad f=\sqrt{1-\frac{2 m}{\tilde{r}}+\frac{C^{2}}{\tilde{r}^{4}}}$. 


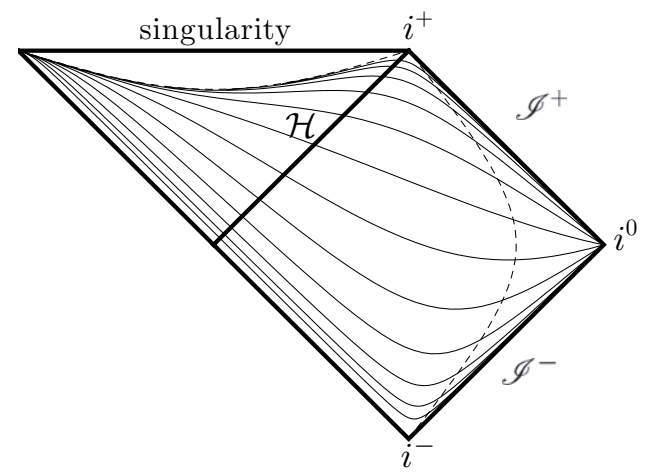

Figure 9. Maximal slices suitable for the moving puncture technique. Dashed lines correspond to $\tilde{r}=\{1.5 m, 4 m\}$.

The standard foliation of Schwarzschild spacetime is a member of this family with $C=0$. The final stationary state for a puncture evolution in Schwarzschild spacetime is similar to a limiting maximal slice with $C=3 \sqrt{3} \mathrm{~m}^{2} / 4$. Figure 9 shows a maximal foliation with $C=3 \sqrt{3} m^{2} / 4$ in the Penrose diagram of the extended Schwarzschild spacetime together with the limiting surface at $\tilde{r}=3 m / 2$, where $f=0$.

\subsection{Hyperboloidal CMC-foliations}

The main difference between the Schwarzschild foliation in figure 7 and the horizon penetrating foliations in figures 8 and 9 is the behavior of surfaces near the event horizon. The intersection at the bifurcation point in Schwarzschild coordinates is not suitable to study the black hole. Similarly, the intersection of surfaces of a foliation at spatial infinity is not suitable to study radiation near null infinity. The construction of hyperboloidal foliations, while geometrically quite different, can in this respect be regarded as similar to the construction of horizon penetrating coordinates.

To find a representation of Schwarzschild spacetime based on hyperboloidal surfaces, we use the general family of spherically symmetric CMC surfaces in the extended Schwarzschild spacetime as discussed in [6, 38] (see [21] for a numerical study). The integration of (14) with $\tilde{K} \neq 0$ and integration constant $C$ leads to the differential equation

$$
h^{\prime}=\frac{\frac{\tilde{K} \tilde{r}^{3}}{3}-C}{\left(1-\frac{2 m}{\tilde{r}}\right) \tilde{P}(\tilde{r})}, \quad \text { with } \quad \widetilde{P}(\tilde{r}):=\sqrt{\left(\frac{\tilde{K} \tilde{r}^{3}}{3}-C\right)^{2}+\left(1-\frac{2 m}{\tilde{r}}\right) \tilde{r}^{4}}
$$

The maximal foliation given in (15) is a member of this family with $\tilde{K}=0$. To get the height function we need to integrate the differential equation in (16). The integral cannot be written in an explicit form but needs to be carried out numerically. The resulting foliation has been plotted in figure 10 .

Scri-fixing conformal compactification as in (4) based on this foliation results in

$$
q=-\left(1-\frac{2 m \Omega}{r}\right) \Omega^{2} \mathrm{~d} t^{2}-\frac{2\left(\tilde{K} r^{3} / 3-C \Omega^{3}\right)}{P(r)} \mathrm{d} t \mathrm{~d} r+\frac{r^{4}}{P^{2}(r)} \mathrm{d} r^{2},
$$

where

$$
P(r):=\Omega^{3} \widetilde{P}(r)=\sqrt{\left(\frac{\tilde{K} r^{3}}{3}-C \Omega^{3}\right)^{2}+\left(1-\frac{2 m(1-r)}{r}\right) \Omega^{2} r^{4}}
$$



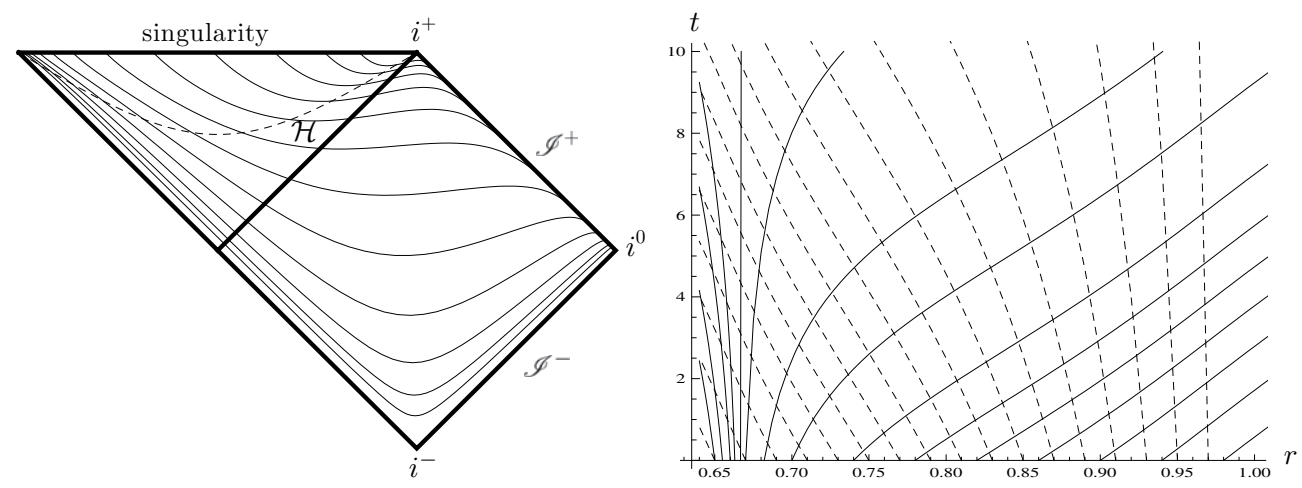

Figure 10. CMC-foliation of the extended Schwarzschild spacetime with $\tilde{K}=0.4, C=2$ and $m=1$. On the left, the Penrose diagram is plotted, on the right in- and outgoing characteristics. The excision surface is taken to be at $\tilde{r}=1.8 \mathrm{~m}$ and is plotted as the dashed line in the Penrose diagram. The event horizon is at $r=2 / 3$.

The global behavior of CMC-surfaces in Schwarzschild spacetime depends on the free parameters as discussed in [38]. The Penrose diagram in figure 10 depicts surfaces which are convenient for numerical calculations. They come from future null infinity, pass the horizon above the bifurcation sphere and run into the future singularity. Depending on the free parameters, $P(r)$ may have a non-vanishing zero set. The corresponding surface lies always inside the event horizon and does not pose a difficulty if the excision surface has been chosen carefully.

The outgoing characteristic speed at $\mathscr{I}^{+}$reads $c_{+}{\mid \mathscr{I}^{+}}=4 \tilde{K}^{2} / 9$, just as for the CMCfoliation of Minkowski spacetime. The main difference to Minkowski spacetime is the black hole region. The coordinate representation of characteristics in figure 10 illustrates that there are no incoming characteristics into the simulation domain both at the excision boundary inside the event horizon and at the outer boundary on future null infinity.

\subsection{Matching}

Schwarzschild spacetime is asymptotically flat in null directions, implying that its asymptotic structure is similar to Minkowski spacetime. We can therefore use the same form of the height function as we did for constructing a hyperboloidal foliation in Minkowski spacetime to get, at least asymptotically, a hyperboloidal foliation in Schwarzschild spacetime. In writing the height function, however, we must be careful about the choice of coordinates. Asymptotic flatness in null directions can be made manifest in terms of the Regge-Wheeler coordinate $\tilde{r}_{*}=r+2 m \ln (r-2 m)$ as described in [44]. It is therefore the height function $h(\tilde{r})=$ $\sqrt{a^{2}+\tilde{r}_{*}^{2}}$, that gives a hyperboloidal foliation in the exterior domain. As the coordinate $\tilde{r}_{*}$ is not suitable near the black hole region, we match the surfaces to interior Eddington-Finkelstein surfaces. The resulting foliation has been plotted in figure 11 .

The simplest method to construct hyperboloidal surfaces seems to be the Taylor expansion of (5) as in (11). In Schwarzschild spacetime, the expansion delivers

$$
\begin{aligned}
h^{\prime}(\tilde{r}) & \sim \frac{\tilde{r}}{\tilde{r}-2 m}\left(1-\left(1-\frac{2 m}{\tilde{r}}\right) \frac{C}{(1+\tilde{r})^{2}}\right)^{\frac{1}{2}} \\
& =1+\frac{2 m}{\tilde{r}}+\frac{8 m^{2}-C}{2 \tilde{r}^{2}}+\frac{C+8 m^{3}}{\tilde{r}^{3}}+O\left(\frac{1}{\tilde{r}^{4}}\right), \quad \text { as } \quad \tilde{r} \rightarrow \infty
\end{aligned}
$$



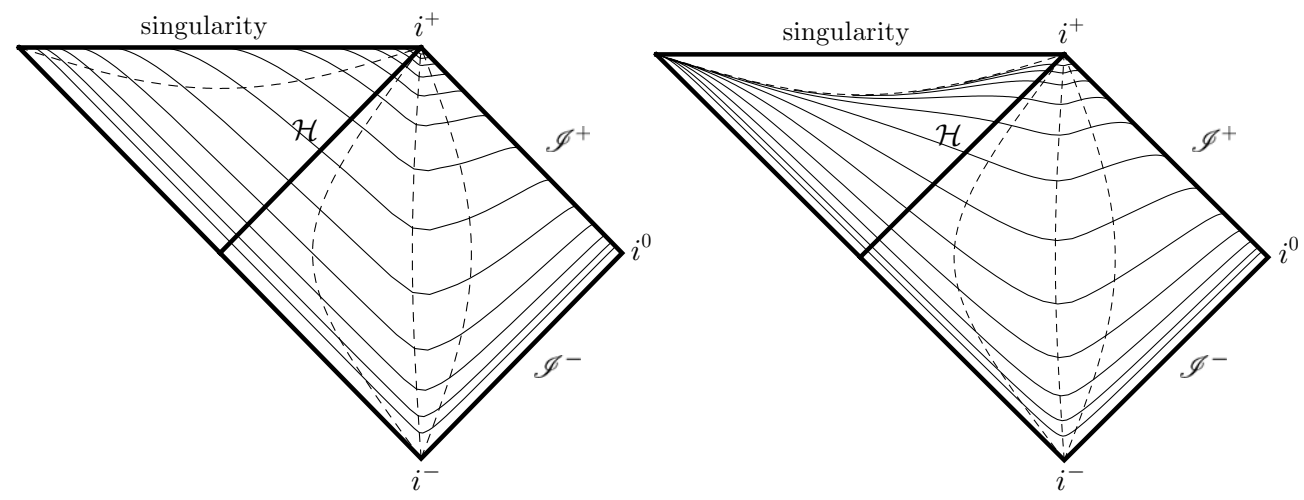

Figure 11. Penrose diagrams of matched foliations in the extended Schwarzschild spacetime. The dashed lines correspond to $\tilde{r}=1.5 \mathrm{~m}, \tilde{r}_{i}=2.1 \mathrm{~m}, \tilde{r}_{m}=2.5 \mathrm{~m}, \tilde{r}_{e}=3 \mathrm{~m}$. The diagram on the left illustrates matching between interior Eddington-Finkelstein surfaces and surfaces with height function $h=\sqrt{9+\tilde{r}_{*}^{2}}$. The diagram on the right illustrates matching between maximal slices and hyperboloidal surfaces with the height function (19).

We take the first two terms so that the height function becomes

$$
h(\tilde{r})=\tilde{r}+2 m \ln \tilde{r},
$$

which essentially corresponds to the Regge-Wheeler coordinate in the exterior domain. Matching to an interior maximal foliation gives the second conformal diagram in figure 11 . The conformally rescaled metric in a scri-fixing gauge in the exterior domain reads

$q=-\left(1-\frac{2 m \Omega}{r}\right) \Omega^{2} \mathrm{~d} t^{2}-2\left(1-\frac{4 m^{2} \Omega^{2}}{r^{2}}\right) \mathrm{d} t \mathrm{~d} r+\frac{8 m^{2}\left(r^{2}-2 m^{2} \Omega^{2}\right)}{r^{3}(r-2 m \Omega)} \mathrm{d} r^{2}$.

The outgoing characteristic speed at future null infinity is $c_{+} \mid \mathscr{I}_{+}=1 /\left(2 m^{2}\right)$. The mean extrinsic curvature of the time slices attains a positive finite value at infinity that depends on the mass of the Schwarzschild spacetime. It reads $\left.\tilde{K}\right|_{\mathscr{I}^{+}}=3 /(2 \sqrt{2} m)$. To gain some control on properties of this asymptotic foliation one can take further terms in the Taylor expansion (18).

\section{Kerr spacetime}

In most studies, going from the spherically symmetric case to the axially symmetric case is a challenging task. Take as an example the construction of suitable null coordinates. Null coordinates can easily be constructed on Minkowski and Schwarzschild spacetimes. In Kerr spacetime, however, they are difficult to deal with and cannot be written in an explicit form $[46,52]$. This is, in part, a consequence of the fact that null coordinates are very rigid. Spacelike foliations on the other hand are simpler to construct due to the large freedom involved in their choices. Further, the property of a spacelike surface to be hyperboloidal does not restrict its form in the interior as we have seen in previous sections. It only determines its asymptotic behavior. This fact in combination with the asymptotic flatness of Kerr spacetime allows us to carry over the construction of a scri-fixing gauge in spherical symmetry directly to axial symmetry on the example of Kerr spacetime.

The most commonly used coordinates for Kerr spacetime in numerical relativity are the Boyer-Lindquist and the Kerr-Schild coordinates. In the following we present the asymptotic 
form of simple hyperboloidal foliations in these coordinates. Other choices are possible and straightforward using the techniques presented so far. The Kerr metrics as given in (21) and (22) can be used in the exterior domain. One may employ the matching technique to obtain a convenient foliation also in the interior depending on the problem at hand.

\subsection{In Boyer-Lindquist coordinates}

The Kerr metric in Boyer-Lindquist coordinates reads

$\tilde{g}=-\left(1-\frac{2 m \tilde{r}}{\widetilde{\Sigma}}\right) \mathrm{d} \tilde{t}^{2}-\frac{4 a m \tilde{r}}{\widetilde{\Sigma}} \sin ^{2} \vartheta \mathrm{d} \tilde{t} \mathrm{~d} \varphi+\frac{\widetilde{\Sigma}}{\widetilde{\triangle}} \mathrm{d} \tilde{r}^{2}+\widetilde{\Sigma} \mathrm{d} \vartheta^{2}+\tilde{R}^{2} \sin ^{2} \vartheta \mathrm{d} \varphi^{2}$,

where

$\widetilde{\Sigma}=\tilde{r}^{2}+a^{2} \cos ^{2} \vartheta, \quad \widetilde{\triangle}=\tilde{r}^{2}+a^{2}-2 m \tilde{r}, \quad \tilde{R}^{2}=\tilde{r}^{2}+a^{2}+\frac{2 m a^{2} \tilde{r} \sin ^{2} \vartheta}{\widetilde{\Sigma}}$.

By $m$ we denote the ADM mass and by $a$ the angular momentum of Kerr spacetime. To choose a suitable height function for a hyperboloidal time coordinate in the exterior domain, we use the Taylor expansion of (5). It reads

$$
h^{\prime}(\tilde{r})=1+\frac{2 m}{\tilde{r}}+\frac{8 m^{2}-C-a^{2} \sin ^{2} \vartheta}{2 \tilde{r}^{2}}+O\left(\frac{1}{\tilde{r}^{3}}\right), \quad \text { as } \quad \tilde{r} \rightarrow \infty .
$$

We use the first two terms and set $h(\tilde{r})=\tilde{r}+2 m \ln \tilde{r}$, as for Schwarzschild spacetime in Schwarzschild coordinates. It is interesting that the height function for a hyperboloidal foliation does not necessarily depend on angular momentum but on mass. On the other hand, the form of the height function should not surprise us, as asymptotically, the Kerr metric in Boyer-Lindquist coordinates approaches the Schwarzschild metric in Schwarzschild coordinates. With the above choice of the height function, the components of the metric in the exterior domain of Kerr spacetime in a scri-fixing gauge take the form

$$
\begin{aligned}
& g_{t t}=-\left(1-\frac{2 m r \Omega}{\Sigma}\right) \Omega^{2}, \quad g_{t r}=-\left(1-\frac{2 m r \Omega}{\Sigma}\right)\left(1+\frac{2 m \Omega}{r}\right), \\
& g_{t \varphi}=-\frac{2 a m r \Omega^{3}}{\Sigma} \sin ^{2} \vartheta, \quad g_{r \varphi}=-\frac{2 a m \Omega}{\Sigma}(r+2 m \Omega) \sin ^{2} \vartheta \\
& g_{r r}=\frac{1}{\Omega^{2}}\left(\left(\frac{2 m \Omega r}{\Sigma}-1\right)\left(\frac{2 m \Omega}{r}+1\right)^{2}+\frac{\Sigma}{\triangle}\right), \quad g_{\vartheta \vartheta}=\Sigma, \quad g_{\varphi \varphi}=R^{2} \sin ^{2} \vartheta
\end{aligned}
$$

where we have set $\Sigma=\Omega^{2} \widetilde{\Sigma}, \triangle=\Omega^{2} \widetilde{\triangle}$ and $R=\Omega \tilde{R}$. A calculation shows that the rescaled metric component $g_{r r}$ is manifestly regular at $\{\Omega=0\}$ and can be written as

$$
\begin{aligned}
g_{r r}=\frac{8 m^{2}}{\Sigma}(1 & \left.+\frac{m \Omega}{r}\right)-\frac{a^{2} \sin ^{2} \vartheta}{\triangle}-\frac{4 m^{2}}{r^{2}} \\
& +\frac{2 m}{r \triangle \Sigma}\left(r^{2}\left(2 m r-a^{2} \Omega\right)-a^{2} \Omega \cos ^{2} \vartheta\left(2 a^{2} \Omega^{2}+r(r-4 m \Omega)\right)\right) .
\end{aligned}
$$

The rescaled metric becomes at future null infinity

$$
g{\mid \mathscr{I}^{+}}=-2 \mathrm{~d} t \mathrm{~d} r+\left(8 m^{2}-a^{2} \sin ^{2} \vartheta\right) \mathrm{d} r^{2}+\mathrm{d} \vartheta^{2}+\sin ^{2} \vartheta \mathrm{d} \varphi^{2} .
$$

We see that, in these coordinates, the coordinate speed of outgoing characteristics depends on $\vartheta$ in a manner related to the angular momentum of the Kerr black hole. 


\subsection{In Kerr-Schild coordinates}

The Kerr metric in ingoing Kerr-Schild coordinates reads

$$
\begin{gathered}
\tilde{g}=-\left(1-\frac{2 m \tilde{r}}{\widetilde{\Sigma}}\right) \mathrm{d} \tilde{t}^{2}+\frac{4 m \tilde{r}}{\widetilde{\Sigma}} \mathrm{d} \tilde{t} \mathrm{~d} \tilde{r}-\frac{4 a m \tilde{r}}{\widetilde{\Sigma}} \sin ^{2} \vartheta \mathrm{d} \tilde{t} \mathrm{~d} \varphi-2 a \sin ^{2} \vartheta\left(1+\frac{2 m \tilde{r}}{\widetilde{\Sigma}}\right) \mathrm{d} \tilde{r} \mathrm{~d} \varphi \\
+\left(1+\frac{2 m \tilde{r}}{\widetilde{\Sigma}}\right) \mathrm{d} \tilde{r}^{2}+\widetilde{\Sigma} \mathrm{d} \vartheta^{2}+\tilde{R}^{2} \sin ^{2} \vartheta \mathrm{d} \varphi^{2},
\end{gathered}
$$

with the definitions (20). For the choice of the height function we use the Taylor expansion of (5) as before and get

$$
h^{\prime}(\tilde{r})=1+\frac{4 m}{\tilde{r}}+\frac{16 m^{2}-C}{2 \tilde{r}^{2}}+O\left(\frac{1}{\tilde{r}^{3}}\right), \quad \text { as } \quad \tilde{r} \rightarrow \infty .
$$

Integrating the first two terms gives $h(\tilde{r})=\tilde{r}+4 m \ln \tilde{r}$. With this choice of the height function, the components of the resulting metric in the exterior domain of Kerr spacetime in a scri-fixing gauge take the form

$$
\begin{aligned}
& g_{t t}=-\left(1-\frac{2 m r \Omega}{\Sigma}\right) \Omega^{2}, \quad g_{t r}=-\left(1+\frac{4 m \Omega}{r}\right)+\frac{4 m \Omega}{\Sigma}(r+2 m \Omega), \\
& g_{t \varphi}=-\frac{2 a m r \Omega^{3}}{\Sigma} \sin ^{2} \vartheta, \quad g_{r \varphi}=-a \sin ^{2} \vartheta\left(1+\frac{4 m \Omega}{\Sigma}(r+2 m \Omega)\right), \\
& g_{r r}=\frac{8 m}{r^{2} \Sigma}(r+2 m \Omega)\left(2 m r-\Omega a^{2} \cos ^{2} \vartheta\right), \quad g_{\vartheta \vartheta}=\Sigma, \quad g_{\varphi \varphi}=R^{2} \sin ^{2} \vartheta .
\end{aligned}
$$

The rescaled metric becomes at future null infinity

$$
g{\mid \mathscr{I}^{+}}=-2 \mathrm{~d} t \mathrm{~d} r+16 m^{2} \mathrm{~d} r^{2}-2 a \sin ^{2} \vartheta \mathrm{d} r \mathrm{~d} \varphi+\mathrm{d} \vartheta^{2}+\sin ^{2} \vartheta \mathrm{d} \varphi^{2} .
$$

\section{Discussion}

It has long been suggested that using a hyperboloidal foliation in combination with conformal compactification of the metric to include null infinity in the computational domain would be a solution to the outer boundary problem as well as the radiation extraction problem within the $3+1$ approach in numerical relativity $[17,32]$. This method requires an adequate choice of coordinate and conformal gauge in the asymptotic domain. An especially suitable class of gauges for numerical studies on radiative properties of fields is scri-fixing $[16,35]$. While it has been known how to construct an explicit scri-fixing gauge in Minkowski spacetime $[34,39]$, there was no such construction available for black hole spacetimes. In this paper we have explicitly constructed and studied scri-fixing gauges in Minkowski, Schwarzschild and Kerr spacetimes. The presented constructions are in such a generality that the methods can be applied to any explicitly given asymptotically flat spacetime admitting a smooth conformal compactification at null infinity.

We may argue that scri-fixing gauges are both natural and useful. Observers with respect to which the coordinate location of $\mathscr{I}^{+}$is independent of time represent asymptotically stationary observers. We have seen in the examples that in the asymptotic limit, Killing observers become null. This is also seen by the fact that a scri-fixing gauge in a stationary spacetime can be constructed such that the metric is manifestly time independent. In this sense, scri-fixing reflects the astrophysical situation that the gravitational wave detectors are asymptotically stationary observers of an isolated system. It is also with respect to such observers that notions of conserved quantities can conveniently be defined. Choosing a scri-fixing gauge 
would considerably simplify the calculation of such quantities and the analysis of numerical solutions. Due to the manifest regularity of the rescaled metric in our construction, points at infinity can be treated on the same level as finite points.

A further suitable property of the foliations we discussed is their intrinsic time asymmetry. Many calculations in numerical relativity are made with time symmetric data. It seems highly unlikely that such data can be regarded as physically reasonable. Hyperboloidal surfaces on the other hand do not allow time symmetry by their very nature and therefore reflect the physical situation more closely.

That an explicit scri-fixing gauge can be useful has been demonstrated in $[14,15]$ in Minkowski spacetime. A natural next step would be to extend such studies to black hole spacetimes and to other test fields. One can also study to what extent the outer boundary introduced in currently common numerical calculations influences the solution. In particular, the matching technique would allow us to make such a comparison conveniently.

The main motivation to include null infinity in the computational domain is to have access to the correct asymptotic waveform. It seems, however, that the approximations commonly used in numerical relativity may be sufficiently accurate for many purposes. An interesting question to study in this context would be whether and to what extent numerical access to null infinity is relevant for astrophysically realistic predictions concerning gravitational radiation signals to be measured on Earth [24]. Just to give an example, it is known that the tail behavior is different at null infinity and at a finite distance away from the source [9, 26, 47]. In this case, a naive interpretation of finite distance extractions may even lead to a misleading error estimate [54]. Certain other features of gravitational radiation might also depend on the observers location in a non-trivial manner.

One may gain some insight from the conformal compactifications presented in this paper for the construction of hyperboloidal initial data [1]. The examples may also serve as a test ground for new ideas on treating the hyperboloidal initial value problem for the Einstein equations. The construction of a scri-fixing gauge in this case can be achieved by prescribing a suitable representation for the conformal factor in terms of coordinates in combination with an appropriate choice of a gauge source function in the general wave reduction of the Einstein equations [56]. Whether this approach can be implemented in numerical calculations remains to be seen. The explicit examples presented in this paper may be useful for performing numerical experiments in this approach.

\section{Acknowledgments}

I am grateful to Helmut Friedrich and Sascha Husa for discussions and comments on the manuscript. I would also like to thank a referee for constructive suggestions.

\section{References}

[1] Andersson L 2002 Lect. Notes Phys. 604183

[2] Bartnik R A and Norton A H 1999 Preprint gr-qc/9904045

[3] Baumgarte T W and Naculich S G 2007 Phys. Rev. D 75067502

[4] Beig R and O'Murchadha N 1998 Phys. Rev. D 574728

[5] Boyle M et al 2007 Phys. Rev. D 76124038

[6] Brill D R, Cavallo J M and Isenberg J A 1980 J. Math. Phys. 212789

[7] Buchman L T and Sarbach O C A 2007 Class. Quantum Grav. 24 S307

[8] Dafermos M and Rodnianski I 2004 Preprint gr-qc/0403034

[9] Dafermos M and Rodnianski I 2005 Invent. Math. 162381

[10] Eddington A S 1924 Nature 113192 
[11] Ehlers J and Krasinski A 2006 Gen. Rel. Grav. 381329

[12] Estabrook F, Wahlquist H, Christensen S, DeWitt B, Smarr L and Tsiang E 1973 Phys. Rev. D 72814

[13] Finkelstein D 1958 Phys. Rev. 110965

[14] Fodor G and Racz I 2004 Phys. Rev. Lett. 92151801

[15] Fodor G and Racz I 2008 Phys. Rev. D 77025019

[16] Frauendiener J 1998 Phys. Rev. D 58064003

[17] Friedrich H 1983 Commun. Math. Phys. 91445

[18] Friedrich H 1986 Commun. Math. Phys. 107587

[19] Friedrich H 2005 Ann. Phys. 1584

[20] Friedrich H and Stewart J 1983 Proc. R. Soc. A 385345

[21] Gentle A P, Holz D E, Kheyfets A, Laguna P, Miller W A and Shoemaker D M 2001 Phys. Rev. D 63064024

[22] Geroch R 1977 Asymptotic Structure of Space-Time ed F Esposito and L Witten (New York: Plenum) pp 1-105

[23] Gómez R, Husa S, Lehner L and Winicour J 2002 Phys. Rev. D 66064019

[24] Gómez R and Winicour J 1992 Phys. Rev. D 452776

[25] Gómez R et al 1998 Phys. Rev. Lett. 803915

[26] Gundlach C, Price R H and Pullin J 1994 Phys. Rev. D 49883

[27] Hannam M, Husa S, Ohme F, Brügmann B and O'Murchadha N 2008 Preprint arXiv:0804.0628

[28] Hannam M, Husa S, O’Murchadha N, Brügmann B, González J A and Sperhake U 2007 J. Phys. Conf. Ser. 66012047

[29] Hannam M, Husa S, Pollney D, Brügmann B and O’Murchadha N 2007 Phys. Rev. Lett. 99241102

[30] Hannam M, Husa S, Sperhake U, Brügmann B and Gonzalez J A 2008 Phys. Rev. D 77044020

[31] Hawking S W and Ellis G F R 1973 The large scale structure of spacetime (Cambridge: Cambridge University Press) ISBN 0-521-09906-4

[32] Hübner P 1998 Preprint gr-qc/9804065

[33] Hübner P 2001 Class. Quantum Grav. 181871

[34] Husa S 2003 Current trends in relativistic astrophysics (Lecture Notes in Physics vol 617) ed L Fernández and L M González (Berlin: Springer) (Preprint gr-qc/0204057)

[35] Husa S, Schneemann C, Vogel T and Zenginoğlu A 2006 AIP Conf. Proc. 841306

[36] Husa S, Zlochower Y, Gomez R and Winicour J 2002 Phys. Rev. D 65084034

[37] Johansen N V and Ravndal F 2006 Gen. Rel. Grav. 38537

[38] Malec E and Murchadha N O 2003 Phys. Rev. D 68124019

[39] Moncrief V 2000 Conformally regular ADM evolution equations Talk at Santa Barbara http://online.itp.ucsb. edu/online/numrel00/moncrief

[40] Moncrief V 2006 The conformally reduced Einstein equations-an alternative approach to Scri Talk at IHP (Paris)

[41] Pazos E, Dorband E N, Nagar A, Palenzuela C, Schnetter E and Tiglio M 2007 Class. Quantum Grav. 24 S341

[42] Penrose R 1963 Phys. Rev. Lett. 1066

[43] Penrose R 1965 Proc. R. Soc. Lond. A 284159

[44] Penrose R 1980 Essays in General Relativity ed F J Tipler (New York: Academic) pp 1-11

[45] Pretorius F 2005 Class. Quantum Grav. 22425

[46] Pretorius F and Israel W 1998 Class. Quantum Grav. 152289

[47] Pürrer M, Husa S and Aichelburg P C 2005 Phys. Rev. D 71104005

[48] Rinne O 2006 Class. Quantum Grav. 236275

[49] Rinne O, Lindblom L and Scheel M A 2007 Class. Quantum Grav. 244053

[50] Sarbach O 2007 J. Phys. Conf. Ser. 91012005

[51] Sarbach O and Tiglio M 2005 J. Hyperbolic Diff. Eqns 2839

[52] Venter L R and Bishop N T 2006 Phys. Rev. D 73084023

[53] Winicour J 2005 Living Rev. Rel. 810 http://www.livingreviews.org/lrr-2005-10

[54] Zenginoglu A 2008 Preprint arXiv:0803.2018

[55] Zenginoğlu A 2007 J. Phys. Conf. Ser. 66012027

[56] Zenginoğlu A 2008 Hyperboloidal evolution with the Einstein equations, in preparation 